\title{
New equipment for neutron scattering cross-section measurements at GELINA
}

\author{
Markus Nyman ${ }^{1, *}$, Thomas Adam ${ }^{2}$, Catalin Borcea ${ }^{3}$, Marian Boromiza $^{3}$, Philippe Dessagne ${ }^{2}$, Gregoire Henning ${ }^{2}$, Maëlle \\ Kerveno $^{2}$, Alexandru Negret $^{3}$, Adina Olacel $^{3}$, Elisa Pirovano ${ }^{4}$, and Arjan Plompen ${ }^{1}$ \\ ${ }^{1}$ European Commission, Joint Research Centre, Unit G.2, Retieseweg 111, 2440 Geel, Belgium \\ ${ }^{2}$ Université de Strasbourg, IPHC, CNRS, UMR7178, 23 Rue du Loess, 67037 Strasbourg, France \\ ${ }^{3}$ Horia Hulubei National Institute of Physics and Nuclear Engineering, 077125 Bucharest-Măgurele, Romania \\ ${ }^{4}$ PTB, Bundesallee 100, 38116 Braunschweig, Germany
}

\begin{abstract}
Two new experimental setups are being developed at European Commission's Joint Research Centre in Geel, Belgium. The scintillator array ELISA (ELastic and Inelastic Scattering Array) is for high-quality neutron scattering cross section and angular distribution measurements. It has the capability to separate neutronand photon-induced events via pulse-shape analysis. Inelastic scattering can also be resolved from the elastic channel. The ELISA setup and data analysis procedure were validated by performing measurements using carbon and iron samples. The DELCO spectrometer (Detection of ELectrons from COnversion) is intended for inelastic neutron scattering cross-section measurements in cases where the detection of $\gamma$ rays is not feasible. The current status of DELCO, results from the first tests, and future prospects will be discussed.
\end{abstract}

\section{Introduction}

Nuclear fission is an important source of energy with very low $\mathrm{CO}_{2}$ emissions. Its main advantage is the high energy density of the fuel, which allows reliable production of large amounts of energy with a small environmental footprint. A number of concerns exist related to the use of nuclear energy, including safety, abundance of uranium, handling of spent fuel, and nuclear proliferation. These issues can be mitigated by developing more advanced systems, such as the Generation IV reactors [1]. These concepts aim at significant improvements in the specific areas of sustainability, safety and reliability, proliferation resistance and physical protection, and economics. To meet these challenges more accurate nuclear data is needed than has previously been available. European Commission's Joint Research Centre (JRC) in Geel, Belgium, is one of the leading producers of experimental neutron data. The present article reports on the development of two new experimental systems at JRC Geel: the scintillator array ELISA (ELastic and Inelastic Scattering Array) and the conversion electron spectrometer DELCO (Detection of ELectrons from COnversion). They will be used in measuring elastic and inelastic neutron scattering cross sections and angular distributions at the GELINA (Geel Electron LINear Accelerator) facility [2, 3]. GELINA is a whiteneutron source designed for measurements using the timeof-flight (TOF) technique. It is capable of simultaneously providing both fast $\left(30 \mathrm{keV}<E_{\mathrm{n}}<20 \mathrm{MeV}\right)$ and moderated $\left(1 \mathrm{meV}<E_{\mathrm{n}}<300 \mathrm{keV}\right)$ pulsed neutron beams to 12 flight paths, ranging in length from $10 \mathrm{~m}$ to $400 \mathrm{~m}$. The

*e-mail: markus.nyman1@ec.europa.eu neutrons are produced by photon-induced reactions from the bremsstrahlung emitted when the electron beam from GELINA impinges on a uranium target. The nominal operating frequency is currently $400 \mathrm{~Hz}$, but previously 800 Hz was used.

\section{ELISA}

ELISA, pictured in Fig. 1, is a spectrometer consisting of 32 liquid organic scintillators. It is designed for measuring elastic neutron scattering cross sections and angular distributions, and can also decouple elastic and inelastic channels if the level density of the nucleus under study is adequate.

\subsection{Detectors and data acquisition}

The experimental setup is described in more detail in Ref. [4]. Half of the detectors have EJ301 (NE-213 equivalent) as the scintillation liquid while the rest have EJ315 $\left(\mathrm{C}_{6} \mathrm{D}_{6}\right)$. The detectors are manufactured by Scionix (models 51A51/2MQOE1-EJ301-NX and 51A51/2MQOE1EJ315-NX). The reason for using both hydrogen and deuterium based scintillators is that it enables a cross check between the two types, which allows the determination of systematic errors during the data analysis phase. A ${ }^{235} \mathrm{U}$ fission chamber is used for measuring the total number of neutrons intercepted by the sample.

The data acquisition for the scintillators is digital, consisting of eight cards with four input channels each, 14bit amplitude resolution and $500 \mathrm{MS} / \mathrm{s}$ sampling rate. The 


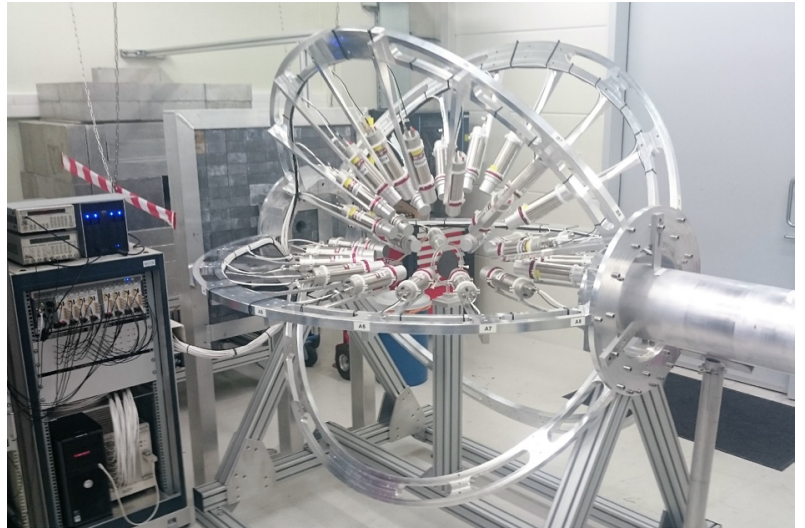

Figure 1. The ELISA spectrometer at GELINA. The fission chamber is located behing the lead wall, upstream of the array. A carbon scattering target is visible at the center.

cards are manufactured by SP Devices (model ADQ14DC4A-VG-PXIe). For synchronization an external $10 \mathrm{MHz}$ reference is provided by a clock generator. The waveforms and the corresponding timestamps are saved for offline processing. The data acquisition for the fission chamber uses conventional analogue electronics.

\subsection{Data analysis}

Analysis of the fission chamber data is described in Ref. [5]. The data analysis procedure for the scintillators is somewhat complex. A full description is available in Ref. [4] and only an overview is given here. The steps of the process are:

- Offline waveform analysis.

- Sorting the events into TOF vs. light-output matrices.

- Background subtraction.

- Correction for multiple neutron scattering.

- Extracting the scattering yields from measured lightoutput distributions.

- Calculating the differential and total integrated scattering cross sections.

Offline analysis for each waveform includes correcting the event timestamp with the constant fraction discriminator (CFD) algorithm, and determining the total integrated charge and the pulse-shape discrimination (PSD) factor. The CFD algorithm is used to improve the time resolution as the initial timestamp is produced by leading-edge triggering. The PSD factor, used for neutron-photon separation, is defined as the ratio between the integral of the tail of the pulse to the total integrated charge. An example can be seen in Fig. 2.

There are two main sources of background: timeindependent room-return neutrons and time-dependent background due to neutrons scattering in air between the end of the flight-path tube and the sample. Room return neutrons can be evaluated from events detected outside the time window containing the GELINA neutron pulse,

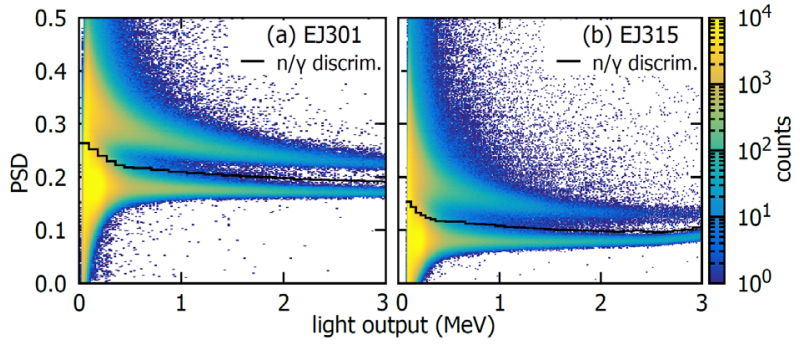

Figure 2. PSD factor as a function of the light output in equivalent electron energy for an EJ301 detector (a) and an EJ315 detector (b). The separation line between neutrons (above) and photons is also displayed. Figure is from [4].

while air scattering is subtracted using a sample out measurement. In experiments at GELINA background from air scattering has been found to be dominant, accounting for up to $30 \%$ of the counts per TOF channel. In practice this necessitates extensive sample out measurements for accurate background subtraction.

For multiple-scattered events the connection between the TOF and incident neutron energy is lost. Correcting for this effect is achieved with an MCNP5 simulation carefully reproducing the experimental setup. The fraction of events arising from multiple scattering is tallied, allowing for a TOF-dependent and detector-dependent correction factor to be determined.

After performing the steps outlined above, one can proceed with the identification of scattering reactions. The kinetic energy of the incident neutrons is obtained from the time-of-flight. The relationship between the TOF, the incident neutron energy $E$, and the energy of the scattered neutron $E^{\prime}$ is

$$
\mathrm{TOF}=\frac{d}{c \sqrt{1-1 /\left(1+\frac{E}{m c^{2}}\right)^{2}}}+\frac{d^{\prime}}{c \sqrt{1-1 /\left(1+\frac{E^{\prime}}{m c^{2}}\right)^{2}}}
$$

where $d$ and $d^{\prime}$ are the distances from the neutron source to the sample and from the sample to the detector, respectively, and $m$ is the neutron mass. The relationship between $E$ and $E^{\prime}$ for a neutron scattering inelastically from a nucleus of mass $M$ is, in the laboratory frame

$$
\begin{aligned}
2 E^{\prime}\left(M c^{2}+m c^{2}\right) & -2 E\left(M c^{2}+m c^{2}\right)+2 E^{\prime} E \\
+ & E^{*}\left(2 M c^{2}+E^{*}\right)=2 c^{2} p p^{\prime} \cos \theta
\end{aligned}
$$

where $\theta$ is the scattering angle, $E^{*}$ the excitation energy of the nucleus, $c p=\sqrt{E\left(E+2 m c^{2}\right)}$, and $c p^{\prime}=$ $\sqrt{E^{\prime}\left(E^{\prime}+2 m c^{2}\right)}$. The incident energy $E$ is solved from Eq. 1 separately for elastic $\left(E^{*}=0\right)$ and inelastic scattering reactions.

The measured light-output distribution for a given TOF bin consists of contributions from elastic scattering and the inelastic scattering reactions which are kinematically possible. Extracting these components is possible after an accurate characterization of the detector's response 
function $R\left(L, E^{\prime}\right)$, which is the probability of a particle with an energy $E^{\prime}$ producing a light signal of intensity $L$. If the particles hitting the detector have an energy distribution $\Phi\left(E^{\prime}\right)$, the corresponding light-output distribution $N(L)$ is

$$
N(L)=\int R\left(L, E^{\prime}\right) \Phi\left(E^{\prime}\right) \mathrm{d} E^{\prime} .
$$

Determining $R\left(L, E^{\prime}\right)$ requires a combination of calibration measurements and Monte Carlo simulations, as thoroughly described in [4]. The experimental response for photons is determined using $\gamma$-emitting calibration sources. For neutrons a TOF measurement with a carbon sample was carried out. The high inelastic threshold of ${ }^{12} \mathrm{C}$ means that below $4.8 \mathrm{MeV}$ incident neutron energy only elastic scattering is possible. Light-output distributions for quasi-monoenergetic neutrons were therefore obtained by selecting a narrow TOF interval, in this case $5 \mathrm{~ns}$ (corresponding to a $20-30 \mathrm{keV}$ energy window). A detailed detector model was implemented into MCNP5 using data provided by the manufacturer. The simulated responses to monoenergetic photons and quasi-monoenergetic neutrons were fitted to the experimental data to obtain the parameters of the detector resolution function and the calibration between integrated charge and light output.

The distribution $\Phi\left(E^{\prime}\right)$ for a given TOF bin, resulting from the neutron beam interacting with the target, can be described as a sum of the various scattering yields, leading to the following expression for the light-output distribution:

$$
N(L)=\varepsilon_{\mathrm{geo}} \sum_{i=0}^{i_{\max }} Y_{\mathrm{sc}, i} R\left(L, E_{i}^{\prime}\right),
$$

where $\varepsilon_{\text {geo }}$ is the geometrical efficiency, $E_{i}^{\prime}$ the energy of a neutron scattered from the $i^{\text {th }}$ excited state $(i=0$ means elastic scattering), $Y_{\mathrm{sc}, i}$ the corresponding scattering yield, and $i_{\max }$ the highest excited level allowed by kinematics. If the response function is known, the scattering yields can be determined by fitting Eq. 4 to the measured light output distribution. The incident neutron energies corresponding to the different $E_{i}^{\prime}$ can then be calculated using Eqs. 2 and 1. The primary quantity extracted from the measured data is the differential scattering cross section

$$
\frac{\mathrm{d} \sigma}{\mathrm{d} \Omega}(E, \theta)=\frac{Y_{\mathrm{sc}}(E, \theta)}{\Delta \Omega N_{\mathrm{n}}(E) n_{\mathrm{S}}},
$$

where $\Delta \Omega$ is the solid angle covered by the detector, $N_{\mathrm{n}}$ the number of neutrons with incident energy $E$ intercepted by the sample, and $n_{\mathrm{S}}$ the areal atom density of the sample. The detector angles $\theta$ are chosen such that their cosines correspond to the zeros of the Legendre polynomial of $8^{\text {th }}$ order. This allows the use of the Gauss-Legendre quadrature method to calculate the total integral cross section as a weighted sum of the differential cross sections.

\subsection{Experimental validation}

Neutron scattering by carbon was chosen as the first test for the ELISA array. It was well suited for this purpose as the cross section is known with an uncertainty of $\lesssim 1 \%$ up to $4.8 \mathrm{MeV}$ neutron energy. Also the differential cross section is a standard for $E_{\mathrm{n}} \leq 1.8 \mathrm{MeV}$. Neutron scattering by iron was chosen as the second experimental case. This was motivated by the uncertainties in the current evaluated nuclear data libraries of iron not meeting the requirements for the development of advanced nuclear systems. Also discrepancies exist between evaluated and experimental cross sections in the fast neutron energy range.

\subsubsection{Neutron scattering by carbon}

The measurement was carried out at GELINA flight path one, 30-m measurement station. The scattering target was a 1-cm thick graphite disk of natural isotopic composition with an areal density of $1.6606(3) \mathrm{g} / \mathrm{cm}^{2}$ and $10-\mathrm{cm}$ diameter. The total integrated elastic scattering cross section from this experiment is shown in Fig. 3 along with previous experimental data included in the EXFOR database. The results from ELISA are compatible with the crosssection standard and other experiments in the range from 1.9 MeV to 7.5 MeV. There are sharp resonances at 2.816 $\mathrm{MeV}$ and $4.937 \mathrm{MeV}$ which remain unresolved with the current energy resolution. To account for this limitation, in Fig. 3 the ENDF/B-VII.1 evaluation is averaged according to the experimental resolution. Some discrepancies between our results and the evaluated cross section can be seen. Above $7.5 \mathrm{MeV}$ the reason is possibly poor statistics, which made the determination of the reaction yields difficult. Below 1.9 MeV the fit of the detector response to the experimental light-output distributions becomes problematic owing to the energy of the scattered neutron being very close to the detection threshold, which leaves only a few data points for the fit.

Four examples of differential cross sections measured at GELINA are compared with other measurements and the ENDF/B-VII.1 evaluation in Fig. 4. The uncertainties on the differential cross section are slightly higher than on the integrated cross section, ranging between $9 \%$ and $15 \%$. In Fig. 4 (a) the evaluation and GELINA data agree very well. At backward and forward angles the agreement with our results is better than with the other datasets. The same is observed, although to a lesser effect, in Fig. 4 (b). At higher energies more differences emerge. In Fig. 4 (c) the different datasets are compatible with each other, but the evaluation does not reproduce them well. In Fig. 4 (d) the GELINA results do not agree with the other available data set angles close to $90^{\circ}$. The multiple-scattering correction can be close to $50 \%$ for the rather thick target used in the present experiment, which could be the cause of difference. A future measurement with a thinner carbon sample is planned to address this issue.

\subsubsection{Neutron scattering by iron}

Two experiments were carried out: one at GELINA and another at the nELBE facility [6] in Helmholtz-Zentrum Dresden-Rossendorf (HZDR), the latter employing a reduced version of ELISA with 16 detectors. The results of these experiments are reported in more detail in Ref. [7]. 

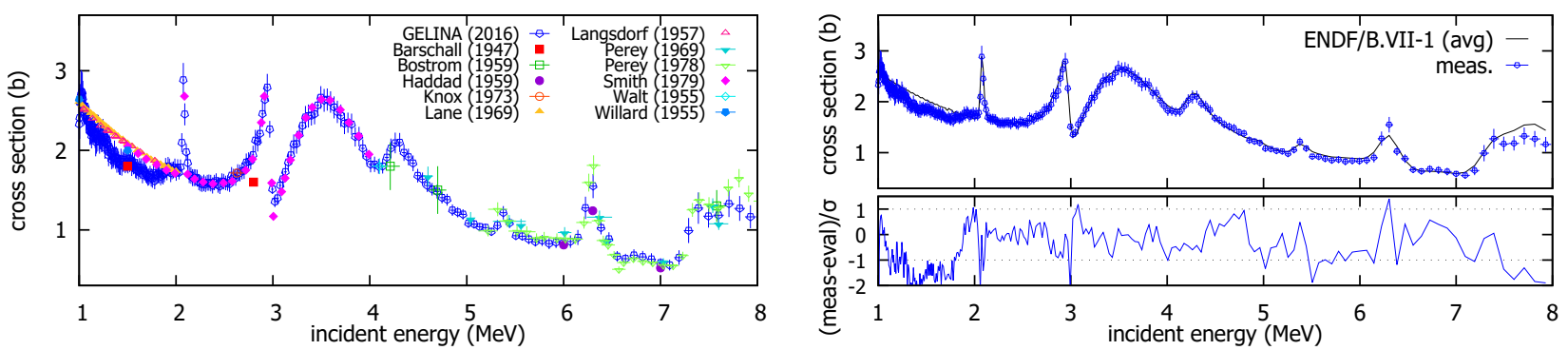

Figure 3. Left: comparison of the total elastic neutron scattering cross section of carbon measured at GELINA with other experiments. Right: Comparison with the ENDF/B-VII.1 evaluation. In the bottom panel the difference between measurement and evaluation divided by the experimental uncertainty (denoted by $\sigma$ ) is shown. Figures are from [4].

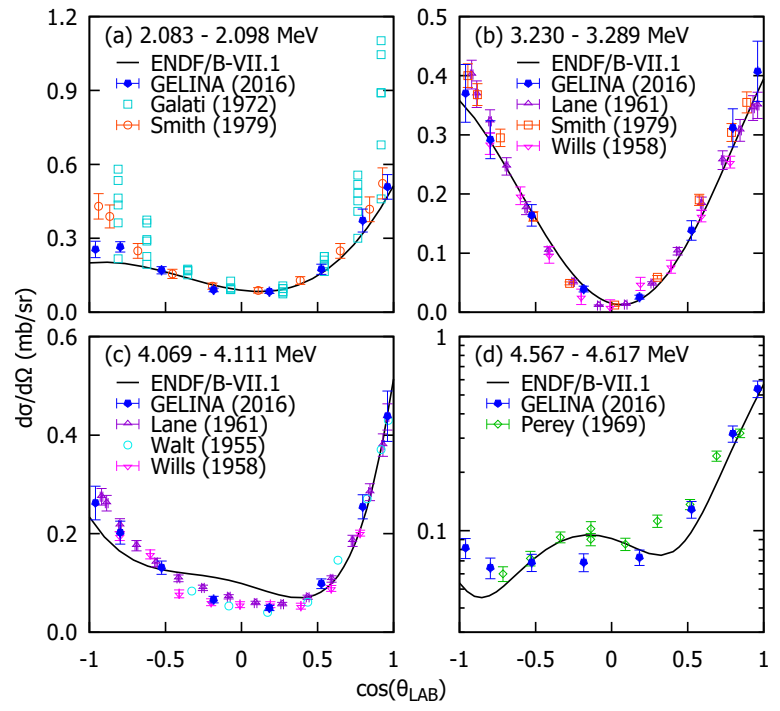

Figure 4. Differential cross section of neutron elastic scattering by carbon as a function of the cosine of the scattering angle in the laboratory frame of reference. The incident neutron energy is indicated in each panel. Comparison with earlier measurements the ENDF/B-VII.1 library are provided. Figures are from [4].

The experimental conditions at GELINA and at nELBE are summarized in Table 1.

To illustrate the capability of separating the elastic and inelastic components, four light-output distributions, measured at GELINA using both EJ301 and EJ315 detectors, are shown in Fig. 5. The incident neutron energy for elastic scattering is $3.31-3.34 \mathrm{MeV}$. The measured data points are compared with the detector responses modeled for elastic scattering and inelastic from the first three levels in ${ }^{56} \mathrm{Fe}(847,2085$, and $2658 \mathrm{keV})$. It was not possible to fit the yield of the third level in Fig. 5 (a) because of low statistics. The different responses of EJ301 and EJ315 detectors are evident. The EJ315 response mainly results from the backward-to-forward asymmetry in the nd scattering, whereas EJ301 has a flatter light-output distribution from the n-p reaction. In the case of EJ315 it is thus simpler to identify the different components, but the model of the detector response is less reliable because of uncertainties in the $n-d$ evaluated differential cross section [8]. The discrepancies between measured and simulated
Table 1. Experimental conditions at GELINA and at nELBE. S $=$ neutron source, $\mathrm{FC}=$ fission chamber, $\mathrm{T}=$ scattering target, and $\mathrm{D}=$ detector. The energy resolution is for $E_{\mathrm{n}}=1 \mathrm{MeV}$.

\begin{tabular}{llll}
\hline & & GELINA & nELBE \\
\hline Sample & mass $(\mathrm{g})$ & $96.215(5)$ & $119.689(1)$ \\
& thickness $(\mathrm{cm})$ & $0.30(1)$ & $0.31(1)$ \\
\multirow{4}{*}{ Flight path } & diameter $(\mathrm{cm})$ & $7.103(1)$ & $7.90(1)$ \\
& $\mathrm{S}-\mathrm{FC}(\mathrm{m})$ & $25.667(5)$ & $6.044(5)$ \\
& $\mathrm{S}-\mathrm{T}(\mathrm{m})$ & $27.037(5)$ & $8.300(5)$ \\
Beam time & $\mathrm{T}-\mathrm{D}(\mathrm{m})$ & $0.300(3)$ & $0.300(3)$ \\
& sample in (h) & 123.8 & 78.4 \\
Avg. flux $\left(10^{4} \mathrm{n} \mathrm{cm}^{-2} \mathrm{~s}^{-1}\right)$ & $2.965(2)$ & $1.076(2)$ \\
Frequency $(\mathrm{Hz})$ & 800 & $1.01 \times 10^{5}$ \\
Time resolution $(\mathrm{ns})$ & 5 & 1 \\
Energy resolution $(\mathrm{keV})$ & 5 & 3 \\
\hline
\end{tabular}

spectra could originate from the evaluated library used in MCNP5 (ENDF/B-VII.1). Modelling of the EJ301 detectors, based on the standard n-p cross section, is more reliable. Overall the simulations are able to reproduce the measured light output distributions well. There are some limitations to separating the elastic and inelastic components. Elastic scattering is forward peaked while inelastic is almost isotropic, and the cross sections have roughly an order of magnitude difference at forward angles. The separation of inelastic scattering is therefore less precise. It is still feasible as illustrated in panels (c) and (d) in Fig. 5, although at higher energies separation is not possible for the most forward angles.

The total integrated cross sections both from measurements at GELINA and at nELBE are shown in Fig. 6 (a). Overall, the GELINA and nELBE data sets agree well with each other, and with previous measurements. The fact that the GELINA and nELBE measurements used a different target, neutron source, and data acquisition system, further increases confidence in the reliability of the ELISA setup and the data analysis procedure. Fig. 6 (b) and (c) show a comparison between the GELINA and nELBE results and the ENDF/B-VIII nuclear data library. In most of the energy range the measurements lie systematically higher than the evaluation. In ENDF/B-VIII the elastic cross section is taken as the difference between the total and the 

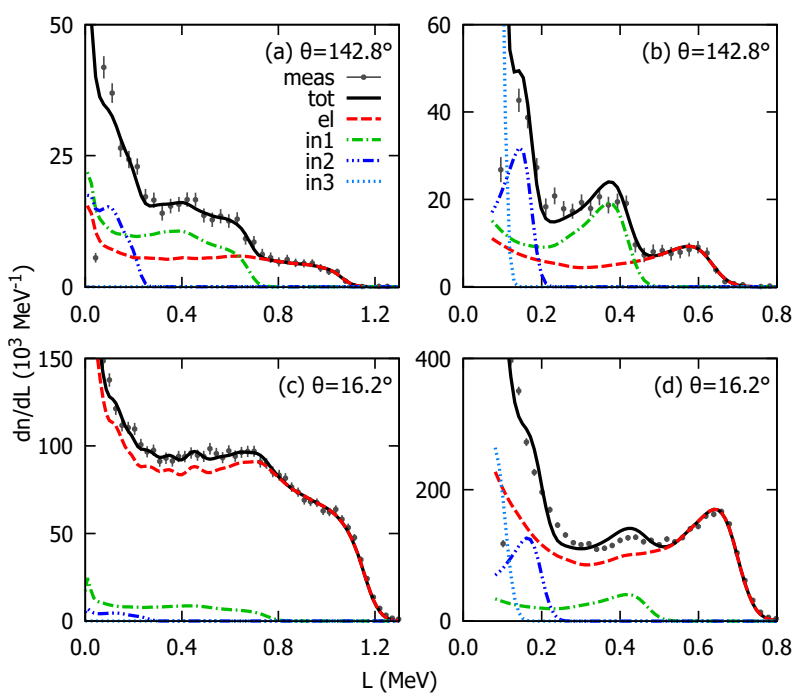

Figure 5. Light-output distributions measured at GELINA with EJ301 (a), (c), and EJ315 (b), (d), detectors at two different scattering angles. The data correspond to $3.31<E_{\mathrm{n}}<3.34 \mathrm{MeV}$ for elastic scattering. Simulated detector response for elastic scattering and inelastic from the first three excited states in ${ }^{56} \mathrm{Fe}$ are shown. Figures are from [7].

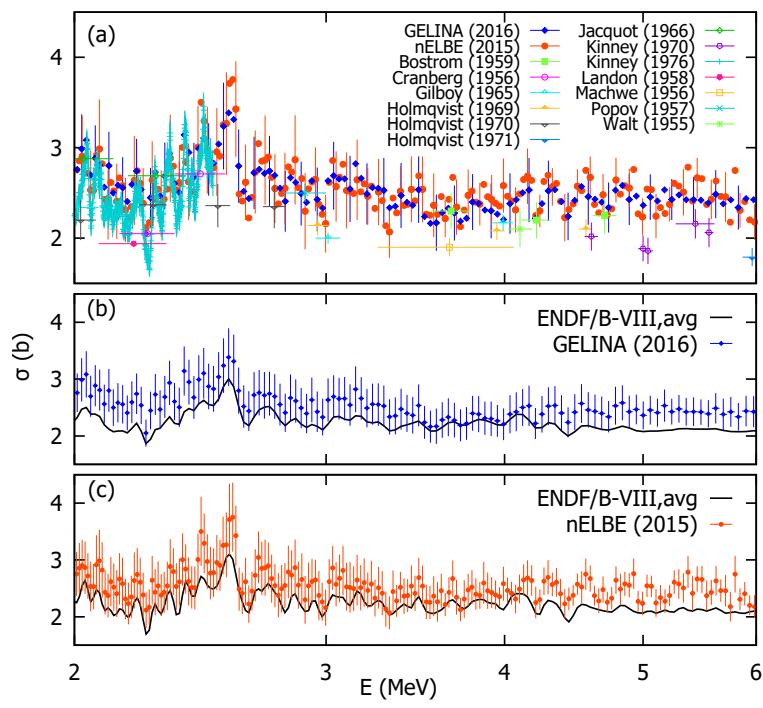

Figure 6. (a) Angle-integrated cross sections of elastic neutron scattering by ${ }^{\text {nat }} \mathrm{Fe}$ from GELINA and nELBE compared with previous experimental data. (b) and (c) Comparison between the GELINA and nELBE results and the ENDF/B-VIII nuclear data library. Figure is from [7].

other partial cross sections, which might be reflected in the discrepancy. Also in Ref. [9] it is suggested that the ENDF elastic cross section should be higher.

\section{DELCO}

Measurements of inelastic neutron scattering cross sections using high-purity germanium (HPGe) detectors have been performed extensively at GELINA using two spectrometers, GAINS and GRAPhEME (see Ref. [10] for

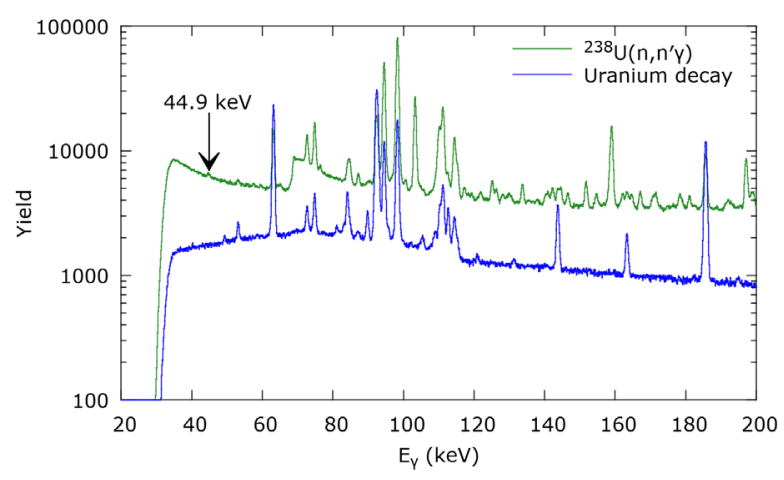

Figure 7. A $\gamma$-ray spectrum from the ${ }^{\text {nat }} \mathrm{U}\left(\mathrm{n}, \mathrm{n}^{\prime} \gamma\right)$ reaction. Background spectrum from the decay of the uranium sample is also shown.

an overview). In some cases, such as highly converted transitions, measuring $\gamma$-production cross sections is not feasible. One example is the first transition in ${ }^{238} \mathrm{U}(\gamma$ ray energy $E_{\gamma}=44.9 \mathrm{keV}$, internal conversion coefficient $\alpha=609$ ). This is illustrated in Fig. 7 where the lowenergy part of a $\gamma$-ray spectrum from the ${ }^{\text {nat }} U\left(n, n^{\prime} \gamma\right)$ reaction, recorded using one of GRAPhEME HPGe detectors, is displayed. A background spectrum from the radioactivity of the sample is also shown. It is obvious from the figure that extracting an accurate $\gamma$-production cross section from the weak 44.9-keV peak on top of a large background is not feasible.

To access transitions like this the electron spectrometer DELCO is being developed. Detection of conversion electrons from $\left(\mathrm{n}, \mathrm{n}^{\prime} \mathrm{e}\right)$ reactions poses its own challenges. Conversion electron spectra are complex, meaning that good energy resolution is crucial. In addition, there will be contributions by electrons from other transitions and the radioactivity of the sample. In the case of the 44.9-keV transition in ${ }^{238} \mathrm{U}$ the L-shell conversion electrons have energies of $24 \mathrm{keV}$ and $28 \mathrm{keV}$, requiring a detection system with low electronic noise. An MCNPX simulation of an electron spectrum from the ${ }^{238} \mathrm{U}\left(\mathrm{n}, \mathrm{n}^{\prime} \mathrm{e}\right)$ reaction, for a detector with a 2.6-keV energy resolution, is depicted in Fig. 8. The detector model used in the simulation is similar to one configuration used in the preliminary DELCO tests.

A drawing of the first version of the DELCO detector chamber is shown in Fig. 9. A scattering target is positioned at $45^{\circ}$ with respect to the neutron beam and four silicon detectors are located on each side, mounted on copper cooling blocks. First test of the setup was performed in this geometry at GELINA. ORTEC ULTRA ion-implanted silicon detectors with an active area of $450 \mathrm{~mm}^{2}, 100 \mu \mathrm{m}$ thickness, and $50 \mathrm{~nm}$ dead layer were used. The detector cooling system was alcohol based. Tests were carried out with several different preamplifiers, measuring the radioactivity from a $99.999 \%{ }^{238} \mathrm{U}$ foil of $80 \mathrm{~mm}$ diameter and $140 \mu \mathrm{g} / \mathrm{cm}^{2}$ areal density. However, sufficient performance for detecting low-energy electrons was not achieved. Subsequently a liquid-nitrogen cooled Si detector with $300 \mu \mathrm{m}$ thickness and $300 \mathrm{~mm}^{2}$ area was used. An energy resolution of $\sim 2.6 \mathrm{keV}$, similar to the one used in 


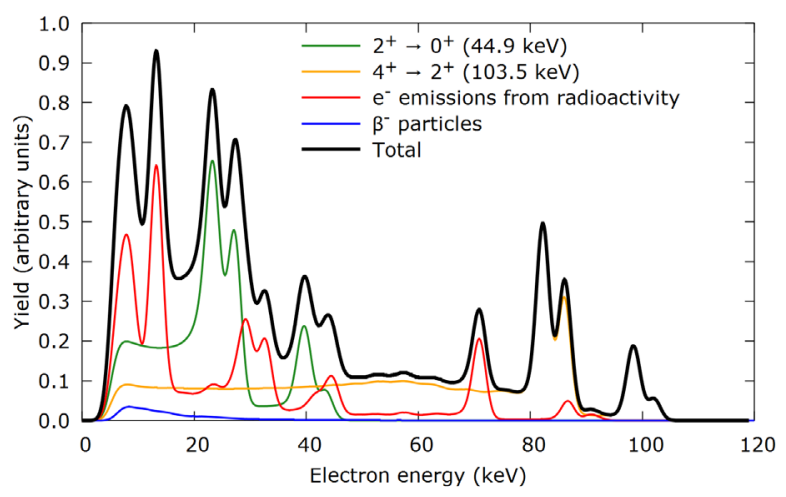

Figure 8. An MCNPX simulation of the response of a Si detector with $2.6-\mathrm{keV}$ energy resolution to electrons from the ${ }^{\text {nat }} U\left(\mathrm{n}, \mathrm{n}^{\prime} \mathrm{e}\right)$ reaction.

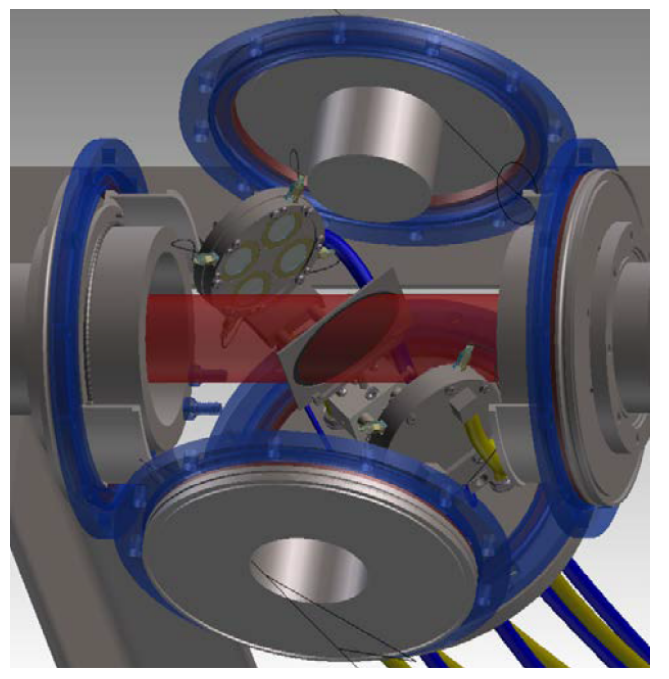

Figure 9. The first version of the DELCO setup.

the simulations presented in Fig. 8, was achieved. It was not possible to detect electrons below $30 \mathrm{keV}$ because the dead layer on the detector turned out to be thicker than expected. Additional tests with the ${ }^{238} \mathrm{U}$ sample in the neutron beam of GELINA were also carried out. Events from interactions between the beam and the target were observed, but further measurements are needed for solid conclusions to be drawn. In the future a new $\mathrm{Si}$ detector manufactured by Mirion Technologies, with an energy resolution of $0.3 \mathrm{keV}$ at $6 \mathrm{keV}$, will be tested.

\section{Conclusions}

Two new experimental setups at JRC Geel for neutron scattering cross section measurements have been intro- duced. The ELISA scintillator array has already been commissioned and is being used for experimental work. The DELCO electron spectrometer is still in the development stage and is not yet ready for cross section measurements.

This work was partially supported by the European Commission through the EUFRAT (EURATOM Contract number FP7211499) and CHANDA (Grant Agreement No. 605203) projects. The authors wish to thank the staff at GELINA, IFIN-HH, and IPHC Strasbourg.

\section{References}

[1] The Generation IV International Forum (GIF), https://www.gen-4.org/gif/jcms/c_9261/ home

[2] A. Bensussan, J.M. Salome, Nucl. Instrum. Methods 155, 11 (1978)

[3] D. Ene, C. Borcea, S. Kopecky, W. Mondelaers, A. Negret, A.J.M. Plompen, Nucl. Instrum. Methods Phys. Res., Sect. A 618, 54 (2010)

[4] E. Pirovano, Ph.D. thesis, Ghent University (2017), IAEA INDC report INDC(BLG)-2, https://www-nds.iaea.org/publications/ indc/indc-blg-2.pdf

[5] C. Rouki, P. Archier, C. Borcea, C. De Saint Jean, J.C. Drohé, S. Kopecky, A. Moens, N. Nankov, A. Negret, G. Noguère et al., Nucl. Instrum. Methods Phys. Res., Sect. A 672, 82 (2012)

[6] E. Altstadt, C. Beckert, H. Freiesleben, V. Galindo, E. Grosse, A.R. Junghans, J. Klug, B. Naumann, S. Schneider, R. Schlenk et al., Ann. Nucl. Energy 34, 36 (2007)

[7] E. Pirovano, R. Beyer, M. Dietz, A.R. Junghans, S.E. Müller, R. Nolte, M. Nyman, A.J.M. Plompen, M. Röder, T. Szücs et al., Phys. Rev. C 99, 024601 (2019)

[8] E. Pirovano, R. Beyer, A.R. Junghans, N. Nankov, R. Nolte, M. Nyman, A.J.M. Plompen, Phys. Rev. C 95, 024601 (2017)

[9] M.T. Wenner, A. Haghighat, J.M. Adams, A.D. Carlson, S.M. Grimes, T.N. Massey, Nucl. Sci. Eng. 170, 207 (2012)

[10] M. Kerveno, A. Bacquias, C. Borcea, P. Dessagne, G. Henning, L.C. Mihailescu, A. Negret, M. Nyman, A. Olacel, A.J.M. Plompen et al., Eur. Phys. J. A 51, 167 (2015) 